\title{
Corela
}

Cognition, représentation, langage

16-1 | 2018

Vol. $16, \mathrm{n}^{\circ} 1$

\section{Je vois ce que tu veux dire}

\section{Jean-Jacques Franckel}

\section{OpenEdition}

\section{Journals}

Édition électronique

URL : http://journals.openedition.org/corela/5819

DOI : 10.4000/corela.5819

ISSN : 1638-573X

\section{Éditeur}

Cercle linguistique du Centre et de l'Ouest - CerLICO

\section{Référence électronique}

Jean-Jacques Franckel, « Je vois ce que tu veux dire », Corela [En ligne], 16-1 | 2018, mis en ligne le 08 juillet 2018, consulté le 07 mai 2019. URL : http://journals.openedition.org/corela/5819 ; DOI : $10.4000 /$ corela.5819

Ce document a été généré automatiquement le 7 mai 2019.

\section{(c) (i) (2)(2)}

Corela - cognition, représentation, langage est mis à disposition selon les termes de la licence Creative Commons Attribution - Pas d'Utilisation Commerciale - Partage dans les Mêmes Conditions 4.0 International. 


\title{
Je vois ce que tu veux dire
}

\author{
Jean-Jacques Franckel
}

1 Cet article ${ }^{1}$ est centré sur une analyse du verbe dire qui va à l'encontre d'une conception classique selon laquelle ce verbe renverrait par lui-même à une activité de parole consistant en une transmission verbale d'un locuteur à un allocutaire. Plusieurs de ses emplois où personne ne parle à quiconque s'avèrent cruciaux pour en comprendre le fonctionnement.

2 Ce verbe présente la particularité de se prêter à plus d'une centaine d'emplois répertoriés comme «locutions» ou expressions figées (cf. en particulier Gomez-Jordana \& Anscombre (2015), Rouanne \& Anscombre (2016)). Nous nous arrêterons à l'une d'entre elles: $T u$ vois ce que je veux dire, en nous interrogeant notamment sur le caractère peu naturel de la séquence ? Je vois ce que tu dis et en défendant l'hypothèse que cette séquence est analysable en termes d'une intrication entre les paramètres constitutifs de l'identité sémantique des verbes voir, vouloir et dire.

\section{CARACTÉRISATION DE DIRE}

3 Notre approche du verbe dire se fonde sur l'observation de la façon dont son sens s'élabore à partir de la diversité de ses emplois dans toute leur diversité et leur complexité, et non sur quelque schéma de communication établi a priori et hors la langue par lequel dire serait de quelque façon associé à la transmission de paroles d'un locuteur à un autre locuteur, conception au demeurant difficilement compatible avec des emplois tels que Ça te dit de venir faire un tour avec moi? Ce nom me dit quelque chose; Quelque chose me dit qu'il va venir ce soir; Il est, disons, un peu décalé; Non mais dis donc, ça va pas la tête ?! ou encore Ça ne me dit rien qui peut signifier d'un côté ça ne m'évoque rien, de l'autre ça ne me tente pas. Notons que ces emplois n'ont pas d'équivalent immédiat dans de nombreuses langues, leur traduction passant par de tout autres verbes. Soulignons également que ces emplois du verbe dire ne sont en rien marginaux ou secondaires, ni du point de vue de leur fréquence d'emploi, ni du point de vue de la dynamique du fonctionnement qu'ils font apparaître ${ }^{2}$. 
4 Nous partirons de la caractérisation suivante ${ }^{3}$ qui vise à la fois à définir l'identité du verbe et à constituer le point de départ d'un raisonnement sur l'organisation de la variation de ses multiples valeurs :

Dire marque qu'une forme en se présentant à un sujet $S$ fait l'objet d'une représentation $\mathrm{R}$ dont $\mathrm{S}$ est le site. la représentation $\mathrm{R}$ (il va venir ce soir) dont elle fait l'objet. forme (quelque chose me dit qu'il va venir ce soir) soit la représentation $\mathrm{R}$ (ce visage me dit quelque chose) n'est pas identifiable en tant que telle et ne peut correspondre qu'à l'indéfini quelque chose (correspondant à une prédication d'existence). Dans le premier cas, $\mathrm{S}$ en étant site d'une représentation $\mathrm{R}$ marque la prédication d'existence d'une forme qui en fait l'objet; dans le second cas, il y a prédication d'existence d'une $\mathrm{R}$ dont $\mathrm{S}$ est le site, et cela du fait qu'une forme préconstruite se présente.

14 - Ça te dit de venir faire un tour? Il s'agit de rendre compte du fait que dire est employé sans aucun complément possible et que ce tour n'apparaît essentiellement qu'à la forme interrogative (ou avec le conditionnel dans le tour assertif : ça me dirait bien d'aller faire un tour) ou encore à la forme négative : non merci, ça ne me dit pas.

2) Dans la seconde série, le sujet syntaxique de dire peut correspondre à un sujet énonciateur (noté $\mathrm{S}_{\mathrm{o}}$ ). Dans ce cas, un sujet $\mathrm{S}_{\mathrm{o}}$ présente une forme à un sujet $\mathrm{S}$ (cette forme faisant l'objet d'une représentation dont S est le site) : Paul m'a dit : va te faire voir ; Paul m'a dit qu'il viendrait. Ces exemples mettent donc en jeu un sujet locuteur (Paul) qui présente une forme (va te faire voir / qu'il viendrait) à $S(m e)$ à valeur d'allocutaire. Il s'agit des emplois " déclaratifs » de dire. La présentation peut prendre toute une série de valeurs (en particulier de « mise en scène ») sur lesquelles nous reviendrons ${ }^{4}$.

Pour comprendre l'identité invariante à laquelle correspond cette caractérisation de dire ainsi que le déploiement des variations dont elle constitue un centre organisateur, il faut passer par l'analyse des mots mis en jeu : représentation dans ses rapports à présentation ainsi que forme. 


\section{PRÉSENTATION / REPRÉSENTATION. PRÉFIXE RE-}

17

18

\subsection{Préfixe re-}

21 Nous partirons de l'analyse de $r^{-5}$ comme préfixe dans le verbe représenter en nous fondant sur l'analyse de Paillard (2006) selon laquelle un verbe préfixé met en jeu une combinatoire entre deux types de relations :

- relation entre les paramètres de la forme schématique (FS) du verbe correspondant à la base ;

23 - relation établie par le préfixe entre deux éléments $\mathrm{X}$ et $\mathrm{Y}$.

$24 \mathrm{X}$ coïncide avec l'un des paramètres de la $\mathrm{FS}(\mathrm{V})$ sur lequel vient donc se greffer la mise en relation établie par le préfixe ; Y correspond à un élément externe à la $\mathrm{FS}(\mathrm{V})$ qui introduit des déterminations sur $\mathrm{X}$.

25 L'hypothèse générale que nous proposons est que re-plonge dans un espace horsactualisation, hors du plan temporel, l'élément $\mathrm{X}$ pris dans une première construction opérée sur le plan temporel en le mettant en relation à $\mathrm{Y}$ qui est défini hors du plan temporel (hors de l'actualisation du prédicat où $X$ est en jeu). Plus précisément: reconfère à $\mathrm{Y}$ le statut de référence de $\mathrm{X}$. Le terme de référence d'une entité $\mathrm{X}$ désigne une entité $\mathrm{Y}$ qui 1) a à voir avec $\mathrm{X}$; 2) est autonome relativement à l'ancrage spatio-temporel de $\mathrm{X}$ (i.e. l'intégration de X dans la relation prédicative telle qu'elle s'actualise); 3) introduit des déterminations sur $X$ indépendantes de l'actualisation du verbe ; 4) fait l'objet d'une appropriation / évaluation subjective (variable : bon, utile, souhaitable, stable, normal, etc.)

$\mathrm{Y}$ en tant que référence de $\mathrm{X}$ confère un statut à l'actualisation de $\mathrm{X}$ indépendamment de cette actualisation. Cette référence constitue la condition ou la circonstance hors du plan temporel de l'actualisation du procès dans lequel $\mathrm{X}$ est mis en jeu. Re-P marque que $\mathrm{P}$ s'actualise (donc dans le plan temporel) en référence à ce qui constitue le repère de cette actualisation hors du plan temporel. Nous donnons quelques exemples à titre d'illustration. Pour chaque verbe, nous proposons une forme schématique de la base permettant d'illustrer et de décrire la combinatoire entre les deux types de relations.

\subsubsection{Relâcher}

27 - Lâcher marque la rupture d'une solidarité établie entre deux éléments $\mathbf{a}$ et $\mathbf{b}$ qui acquièrent une autonomie à travers cette désolidarisation (cette autonomie ne se définit que relativement à une non autonomie ou une solidarité première).

$\mathbf{a}$ est localisé par $\mathbf{b}$ dont $\mathbf{a}$ se désolidarise. L'élément a correspond au complément direct, noté $\mathrm{C}_{1}$ (Paul a lâché ses chiens) ou au sujet syntaxique noté $\mathrm{C}_{0}$ (Le piston a lâché). 
29 - Relâcher confère un double statut à $\mathbf{a}$ : a correspondant à X dans le cadre de la relation XRY marquée par re- est repéré par un site (Y) qui lui confère une localisation autonome, localisation de référence, indépendante de l'actualisation de sa désolidarisation actualisée par rapport à $\mathbf{b}$. Le repérage de $\mathbf{a}(\mathrm{X})$ par le localisateur de référence $\mathrm{Y}$ confère à $\mathbf{a}$ un statut indépendamment de l'actualisation de la désolidarisation de a. Il y a une autonomie de a indépendamment de l'actualisation de son autonomisation.

30 C'est l'autonomie première, originelle, hors du plan temporel, de a qui fonde la solidarité de $\mathbf{a}$ et $\mathbf{b}$ et relâcher marque le retour à cette autonomie originelle (l'une des figures du retour qui est l'une des figures du fonctionnement de re-).

31 L'autonomie de a est d'un côté seconde (sur le plan de l'actualisation du verbe) en tant qu'elle se définit relativement à une solidarité première de $\mathbf{a}$ et $\mathbf{b}$; d'un autre côté première (hors du plan temporel) en tant relative à une référence autonome par rapport à l'actualisation du procès : a est « libre » en tant que libéré et en même temps libre en tant que la liberté relève d'une condition « originelle », indépendante de l'actualisation $\mathrm{du}$ procès de désolidarisation. Relâcher un oiseau $\left(\mathrm{C}_{1}=\mathbf{a}=\mathrm{X}\right)$ marque la libération d'un oiseau dont la condition originelle est d'être libre. $\mathrm{a}=\mathrm{X}$ correspond toujours à $\mathrm{C}_{1}$.

\subsubsection{Rejoindre}

32 - Joindre marque que deux entités $\mathbf{a}$ et $\mathbf{b}$ font en un instant $\mathbf{t}$ l'objet d'une localisation réciproque relevant d'une visée ( $\mathbf{a}$ et $\mathbf{b}$ entrent en contact dans le cadre d'une téléonomie). En dehors de l'instant $t$, il y a séparation première de $\mathbf{a}$ et $\mathbf{b}$ qui est la norme, l'état stable, originel : $\mathbf{a}$ et $\mathbf{b}$ ne "vont pas normalement ensemble » : joindre l'utile et / $\grave{a}$ l'agréable, les deux bouts. $C_{1}$ correspond à l'un des éléments (a) de cette localisation réciproque.

33 Rejoindre met en jeu un localisateur externe (hors du plan temporel) site de la localisation réciproque de $\mathbf{a}$ et $\mathbf{b}$. Les paramètres $\mathbf{a}$ et $\mathbf{b}$ ont pour référence un localisateur commun externe $(\mathrm{Y})$ à leur localisation réciproque : Rejoindre $\mathrm{C}_{1}$ quelque part (ce qui associe le contact à une spatialisation J'ai joint Paul à Paris (quand je / Paul était à Paris) / je vous rejoins sur ce point ; je vous rejoins tout de suite (là où vous êtes) : Je joins Paul au téléphone mais je le rejoins à la cabine téléphonique. Le contact est à la fois instable (relativement à joindre il n'a de statut qu'en un instant $t$ ) et stable en tant que $\mathbf{b}$ correspond à une référence autonome.

\subsubsection{Ressentir}

34 Sentir marque la manifestation de quelque chose (q) qui fait l'objet d'une représentation $\mathrm{R}$ (dont $\mathrm{S}$ est le site) de ce qu'est / comment est ce quelque chose. L'élément q correspond à $C_{1}$. On peut distinguer trois variations selon que $q$ est premier, $R$ première ou que $q$ et $R$ en bloc.

Ressentir, à la différence de sentir qui peut être de l'ordre de la perception, est de l'ordre d'une manifestation interne. Le préfixe re-confère à qun double statut :

36 - dans le cadre de l'actualisation du V, q correspond à ce dont la manifestation dans le temps fait l'objet d'une représentation $\mathrm{R}$;

37 - dans le cadre du repérage par $\mathrm{Y}, \mathbf{q}$ correspond à $\mathrm{X}$ repéré par un élément $\mathrm{Y}$ associé par lui-même à une manifestation indépendante de celle qu'actualise le V. Cette manifestation 
est réduite à sa dimension notionnelle, déconnectée du hic et nunc, du plan temporel, du réel, ce qui renvoie à la valeur de manifestation interne.

\subsection{Représenter / Représentation}

Comme noté, présenter signifie rendre présente dans son identité une entité a à / pour un sujet $\mathrm{S}$ (elle est donnée à voir). Le préfixe re- dans représenter confère un double statut à $\mathbf{a}$. En même temps que a est rendu présent pour $S$ dans un temps présent actualisé, a prend le statut de $\mathrm{X}$ en tant qu'il fait l'objet d'un repérage par un terme $\mathrm{Y}$ investi (en tant que référence) d'une présence indépendante de la présence actualisée marquée par le procès. Cette présence relève d'une construction hors du plan temporel, d'une construction subjective (dont le site est S / dans la position S). Ces deux formes de présence (l'une actualisée, l'autre dont le site est $\mathrm{S}$ ) ont à voir et sont en même temps irréductibles l'une à l'autre (on plonge le présent actualisé dans un espace hors-actualisation, un espace subjectif). L'unité correspondant à a $(\mathrm{X})$ est présente indépendamment de sa présence effective dans le temps et l'espace (il s'agit donc d'une présence à / pour un sujet). Représenter, c'est donner un autre mode de présence que celle qui s'inscrit dans le hic et nunc de cette présence.

Avec le nominal représentation ${ }^{6}, Y$ est introduit par la préposition de (une représentation est une représentation de quelque chose). Du fait de son statut de référence, $\mathrm{Y}$ est investi de valeurs relevant de valuations subjectives, renvoyant en particulier à différentes modalités d'appropriation (ce que S peut / veut faire avec cette représentation)7.

\section{FORME}

\subsection{Caractérisation de forme} $\mathrm{Rf}$ et présentation sont données en bloc. 


\subsubsection{Rf première}

Rf prend valeur de «modèle » relativement auquel la façon dont X se présente pour un sujet se définit, dans des rapports d'ajustement, d'adaptation, de "conformité ». La présentation s'actualise en fonction de Rf. Forme prend le statut de support du conforme.

Mettre un article en forme : présenter l'article en coïncidence avec la représentation de référence de ce qu'est un article.

Une visite pour la forme: une visite dont la présentation entre en coïncidence avec la représentation de référence de qu'est une visite, invariante sous toutes les déterminations possibles de chaque visite singulière. La visite en question n'a d'autre contenu que ce qui lui permet de relever de cette invariance.

\subsubsection{Présentation première}

La façon dont $\mathrm{X}$ se présente est mise en regard d'une Rf.

Cette mise en rapport peut marquer que $\mathrm{X}$ se trouve appartenir à une classe de $\mathrm{X}$ qui ont la même Rf ou que la façon dont $\mathrm{X}$ se présente entre dans une comparaison à Rf.

\subsubsection{X appartient à une classe de $X$ rapportables à la même $R f$}

La grippe aviaire est une forme de grippe très dangereuse.

Grippe aviaire fait partie de la classe des grippes relevant de la même $\mathrm{Rf}$ (grippe dangereuse). Il existe la même différence entre La grippe aviaire est une forme de grippe très dangereuse et $L a$ grippe aviaire est une grippe très dangereuse qu'entre une table de forme ovale (la table appartient à la classe des objets qui ont cette forme, cette Rf) et une table ovale.

Ce médicament est vendu sous forme de comprimés (comparer avec en comprimés)

Ce médicament a comprimé pour forme (Rf.). Ce médicament fait partie de la classe des médicaments qui se présentent relativement à la Rf être comprimé.

\subsubsection{La façon dont $X$ se présente entre dans une comparaison à $R f$}

Il a un visage en forme de poire.

La façon dont son visage se présente peut être mis en relation avec (évoque) une forme représentation de référence Rf.

6 Le jeu du gendarme où il s'agit de reconnaître la forme du gendarme dans le dessin des frondaisons. Il s'agit de discerner dans le dessin la forme caractéristique des attributs du gendarme : je vois le gendarme dans les frondaisons signifie que dans les frondaisons je vois une forme de gendarme / la forme d'un (du) gendarme. La frondaison se présente en évoquant la Rf être gendarme.

\subsubsection{Rf et présentation de $X$ sont données en bloc}

57 La présentation de $\mathrm{X}$ constitue sa propre représentation de référence. On peut distinguer deux cas :

58 - cas de prédication d'existence de l'objet d'une représentation qui est à elle-même sa propre Rf; 
Je vois une forme bizarre se détacher sur le mur.

Vers le soir, nous commençons à distinguer des formes vagues qui se dessinent derrière le voile de brume.

- cas où présentation de $\mathrm{X}$ et $\mathrm{Rf}$ sont construites en bloc dans un jeu d'ajustement qualitatif.

Paul est en (grande, petite, bonne) forme.

Forme est d'un côté la forme correspondant à l'état de Paul telle que cette forme se présente et d'un autre côté la référence de être en forme, correspondant à l'état de meilleure forme possible, avec des rapports d'adéquation variables.

\section{Retour à dire}

\subsection{Rapports spécifiques à dire entre forme et représentation pour un sujet $S$}

Rappelons notre caractérisation : dire marque qu'une forme en se présentant à un sujet $\mathrm{S}$ fait l'objet d'une représentation $R$ dont $S$ est le site.

Nous noterons Rs la représentation dont un sujet $\mathrm{S}$ est le site du fait de la présentation de la forme-représentation de référence Rf. Dire marque que ce qui, en se présentant à un sujet $\mathrm{S}$, fait l'objet pour $\mathrm{S}$ d'une représentation est une forme, et donc une représentation de référence (Rf). Représentation inhérente à une forme (Rf) et représentation pour un sujet (Rs) n'ont de statut que l'une relativement à l'autre, du point de vue de leur construction (détermination que nous notons QNT, renvoyant à une prédication d'existence) comme de leur détermination qualitative (que nous notons QLT).

En tant que représentation de référence, $R f$ est invariante. $R f$ et Rs ne sont pas réductibles l'une à l'autre (une référence n'est pas réductible à ce dont elle est la référence) et entrent dans des rapports variables qui sous-tendent les variations des valeurs de dire.

Il y a un écart irréductible lorsque le rapport entre Rf et Rs est d'ordre purement QNT (l'enjeu étant la prédication d'existence soit de Rf, soit de Rs), ce qui correspond à deux cas: 1) la seule détermination de Rf est le fait même qu'elle se présente, cette présentation correspondant à sa prédication d'existence (quelque chose me dit qu'il va venir ) ; 2) la détermination de Rs est réduite au seul fait que $\mathrm{S}$ est construit comme en étant le site dès lors que Rf se présente (ce visage me dit quelque chose). Dans chacun de ces cas, quelque chose marque cette irréductibilité entre Rf et Rs qui se traduit par une "opacité ", la mise en jeu relativement à un pôle ou à l'autre de « je ne sais pas quoi ».

68 En revanche, le rapport entre Rf et Rs est qualitatif (QLT) dans les emplois " déclaratifs » de dire, où intervient une instance de présentation (qui est l'instance d'actualisation de la présentation) : il m'a dit merci, il m'a dit d'aller me faire voir, il m'a dit que Paul venait

La représentation inhérente à la forme qui fait ainsi l'objet d'une présentation peut entrer dans des rapports de correspondance qualitatifs variables avec la représentation dont $\mathrm{S}$ est le site. Comme nous allons le voir, le rapport qualitatif est susceptible de correspondre à une coïncidence (en particulier dans le cas où dire introduit un « discours direct » mais aussi à des ajustements qualitatifs, qui peuvent en particulier s'inscrire dans des jeux de reformulation (je ne vois pas ce que tu veux dire) ${ }^{9}$. Dans ces emplois, se joue la distance entre présentation et représentation dont $\mathrm{S}$ est le site, distance propre à 
« fragmenter » le rapport à Rf. Ceci est à mettre en relation avec le fait très général (et qui excède dire en tant que tel) que le sens d'un texte ou d'un mot ou d'une parole (Rf) est d'un côté invariant (un mot ou un texte dit ce qu'il dit) comme toute forme, mais de l'autre, c'est-à-dire pour qui le comprend ou l'interprète (S), variable d'un sujet à l'autre, d'un moment à l'autre, d'une époque à l'autre.

Ces deux types de rapport sont précisés et développés dans les variations de dire.

\subsection{Variations}

71 Trois ordres de variations, développées et illustrées dans les paragraphes suivants, se croisent dans les emplois de dire :

72 1) Une première variation (déjà évoquée dans l'introduction) concerne le mode de présentation de Rf qui peut correspondre aux deux cas suivants :

73 a) la présentation de Rf est de l'ordre d'une manifestation dans le temps : dire marque qu'il y a présentation d'une forme dans le temps, sans que cette forme soit présentée par un sujet. Il s'agit d'une manifestation « impersonnelle » (une forme se manifeste d'elle-même), cette manifestation d'une forme étant associée à la construction d'une Rs. On distingue trois sous-cas : la présentation d'une forme dans le temps correspond à la prédication d'existence, soit de cette forme, soit de Rs, soit des deux en bloc;

b) la présentation de Rf est opérée par une instance de présentation (le plus souvent, mais non nécessairement, un sujet énonciateur $\mathrm{S}_{\mathrm{o}}$ ) correspondant au sujet syntaxique $\mathrm{C}_{\mathrm{o}}$. Cette présentation est dissociée de la prédication d'existence de Rf.

75 2) La deuxième variation concerne le mode de construction de $\mathrm{Rf}$ et de Rs qui peuvent être ou non préconstruits. Cette variation ne concerne que les cas où la présentation de Rf est opérée par une instance de présentation (dans les autres cas, Rf et Rs ne sont pas préconstruits).

76 3) La troisième variation concerne la présence effective d'un terme correspondant à l'allocutaire S. Cette présence peut être nécessaire (quelque chose me dit qu'il va venir ce soir ; je te dis mon amitié), possible (je vais (te) dire ce qui s'est passé; je (te) dis des bêtises) ou fortement contrainte, voire impossible (cela va sans dire, dire la messe).

\subsubsection{Première variation : mode de présentation de $\mathrm{Rf}$, soit dans le temps (sans sujet), soit par un sujet.}

77 Ces deux cas correspondent à des modes de construction différents de Rf et de Rs.

\subsubsection{La présentation d'une forme est de l'ordre d'une manifestation dans le temps de cette forme}

Dire marque qu'il y a présentation d'une forme dans le temps et sans sujet (manifestation « impersonnelle » : une forme se manifeste / se présente). De cette manifestation s'ensuit une prédication d'existence soit de cette forme, soit de Rs, soit des deux en bloc.

\subsection{Prédication d'existence d'une forme à travers sa manifestation}

Ce cas correspond à l'exemple : Quelque chose me dit qu'il va venir ce soir ${ }^{10}$. 

pour $\mathrm{S}$ tient à ce qu'une forme se présente, sans aucune autre détermination qualitative que le fait de se présenter qui correspond à sa prédication d'existence (il y a quelque chose qui me dit $<. . .>$, quelque chose qui constitue la référence de la représentation dont $S$ correspondant à me est le site). La dissociation entre les deux constructions est mise en évidence dans la variante : il va venir, quelque chose me le dit : me est siège d'une Rs, et cela du fait que quelque chose (Rf) se présente ${ }^{11}$.

81 La forme n'est pas préconstruite. Elle fait l'objet d'une prédication d'existence (détermination QNT) à travers le fait qu'elle se présente. La détermination qualitative de Rs provient exclusivement de S qui en est le site. Mais dire marque que S n'est site de cette représentation Rs (dont $S$ est donc la seule source de détermination qualitative) que dans la mesure où une forme $\mathrm{Rf}$ se présente. Rf n'ayant d'autre détermination qualitative que le seul fait de sa présentation qui correspond à sa prédication d'existence, le seul $C_{0}$ possible est alors quelque chose.

\subsection{Prédication d'existence de Rs du fait qu'une $R f$ préconstruite se présente}

Ce cas correspond à l'exemple : Cette image / cette histoire / ce visage me dit quelque chose.

Dans le cas précédent la détermination QLT (indéterminée) ne tenait qu'à Rs ; dans ce cas elle ne tient qu'à Rf. La détermination qualitative de Rs est réduite au seul fait que Rf en est l'objet en se présentant. Dans le présent cas, la détermination qualitative de Rs ne peut être autre que quelque chose. ${ }^{12}$

Un cas différent par ailleurs, mais relevant de ce même type de rapport Rf-Rs se trouve illustré par l'exemple : On dirait un lion. On voit une forme (quelque chose dont on ne sait pas de quoi cette chose est forme, ou dont on sait qu'elle n'est pas celle d'un lion). Ce qui se présente (en l'occurrence ce qu'on voit) est ici encore la Rf d'une représentation qualitativement indéterminée, de quelque chose. Par le biais du conditionnel, ce quelque chose est identifié à ce qui pourrait être.

\subsection{Prédication d'existence de Rf et Rs en bloc}

\section{Ça te dit de venir faire un tour?}

$\mathrm{Rf}$ et Rs font l'objet d'une prédication d'existence en bloc relativement à $\mathrm{S}$.

La forme <venir faire un tour> est présentée comme enjeu d'une prédication d'existence pour S. Il s'agit d'un tour essentiellement interrogatif ${ }^{13}$ qui dissocie la préconstruction d'une forme de sa présentation comme non préconstruite pour $\mathrm{S}$ à travers sa reprise par ça. Ça renvoie à l'actualisation, dans le cadre de l'énonciation, de la mention de ce dont il s'agit dans toutes ses composantes en bloc, sans ancrage situationnel, sans détermination QNT. Ça confère à ce à quoi il renvoie un mode de présence qui n'a d'autre détermination que celle que lui confère l'actualisation de son énonciation. La question consiste à savoir s'il y a dire pour toi, c'est-à-dire présentation d'une forme (<venir faire un tour>) telle que tu sois site de Rs de cette forme. Cette forme est d'un côté construite et présentée par le questionneur mais n'est pas directement présentée à $\mathrm{S}$. Du point de vue de $\mathrm{S}$, la forme se présente (ou non). Il y a rupture entre la présentation de Rf par le questionneur et la présentation de Rs pour $\mathrm{S}$ qui n'est pas assimilable à un allocutaire $\left(\mathrm{S}_{\mathrm{o}}\right.$ ne « dit " pas 
quelque chose à S). Il ne s'agit donc pas d'un cas de transmission de paroles, comme dans la variation suivante (4.2.2.).

Reste à expliquer le caractère d'invitation que prend ce tour et la parenté de dire avec tenter. Dire ne peut guère introduire une séquence à caractère détrimental ou désagréable :? Ça te dit de devoir repasser cet examen? Dans ce dernier cas la séquence $<$ repasser ton examen> a un ancrage situationnel (il en est question dans le contexte), elle s'appuie sur une première construction, elle a par conséquent une détermination QNT. En revanche, <venir faire un tour> correspond à une proposition ex abrupto : sa construction coïncide avec sa présentation et ne tire sa positivité que de sa seule détermination qualitative, qui ne peut être associée qu'à une valuation positive. De plus, du fait que $S_{\text {o }}$ présente Rf à $\mathrm{S}$ (relativement auquel de son côté Rs se présente ou non), Rs fait que la séquence introduite par dire tend à relever d'une activité conjointe du questionneur et de S.

Dans les trois cas de 4.2.1.1 où l'enjeu est la seule existence réciproque de Rf et de Rs, il y a coupure qualitative de l'une par rapport à l'autre. La détermination QLT de l'une va de pair avec l'indétermination QLT de l'autre. Le rapport entre Rf et Rs est un rapport d'existence (QNT) et non un rapport qualitatif (QLT).

\subsubsection{4. Ça ne me dit rien}

La forme négative ça ne me dit rien peut renvoyer à deux des cas précédemment distingués :

91 - dans un cas rien s'oppose à quelque chose et renvoie au cas 4.2.1.1.2. illustré par ce visage me dit quelque chose;

92 - dans le second cas rien peut être remplacé par pas et marque que S ne se constitue pas comme site de Rs construit en bloc avec Rf, avec la valeur Ça ne me tente pas.

93 Ça ne me dit rien qui vaille peut s'analyser comme relevant de ces deux cas à la fois. D'un côté, rien s'oppose à quelque chose, à l'opacité de ce quelque chose, le quelque chose que ça me dit étant valué négativement (Rs ne "vaut rien»); d'un autre côté, rien qui vaille correspond à la version négative de tenter. Ça ne me tente pas, ça ne me plaît pas (justifié par le fait qu'en même temps le quelque chose que ça me dit est valué négativement). Je ne sais pas trop ce que ça me dit, mais ce que ça me dit ne me dit pas (plait pas / tente pas) :

«Ce bloc de farine ne me dit rien qui vaille ». (La Fontaine, Le chat et un vieux Rat).

Voulez-vous que je vous dise, ce qu'ils nous disent ne me dit rien qui vaille $»^{14}$

\subsubsection{Présentation à $S$ de $R f$ par une instance qui présente.}

Cette instance qui présente $\mathrm{Rf}$ est en général un sujet $\mathrm{S}_{\mathrm{o}}$ correspondant au sujet syntaxique $C_{o}$, mais nous verrons que d'autres cas sont possibles. Nous distinguerons deux cas selon que la forme Rf est préconstruite ou non. Le premier cas correspond essentiellement aux emplois « déclaratifs » de dire et recouvre toute une variété de modes de présentation possibles de Rf par l'instance qui présente, allant de la neutralité de cette présentation (dire comme introducteur de "discours direct " à différentes sortes de " mises en scène " énonciatives ; dans le second cas, l'instance (le sujet énonciateur $\mathrm{S}_{\mathrm{o}}$ ) construit Rf, ce qui rend possibles des jeux d'ajustement entre Rf et Rs. Ces deux cas correspondent au second ordre de variation que nous examinons dans le $\S$ suivant. 


\subsubsection{Deuxième variation : $\mathrm{Rf}$ préconstruite ou non}

\subsubsection{1. $R f$ est préconstruite}

97 La présentation de Rf est dissociée de sa prédication d'existence. Rf peut correspondre à une forme "toute faite»: dire la messe, dire zut à quelqu'un, dire des mots d'amour; ou à un discours que l'instance qui présente « rapporte », comme le marque l'expression discours rapporté, discours direct ou indirect: Paul m'a dit qu'il viendrait. Dire peut notamment être employé dans des contextes consistant à

98 - rendre publique une parole (avec des effets plus ou moins ostentatoires) : je (vous) le dis haut et fort ; [...]; en vérité, je vous le dis [...], cette présentation pouvant s'inscrire dans un cadre social ou un rituel formaté ;

99 - conférer une valeur d'engagement : quand je dis que j'y vais, j'y vais ; c'est dit! Tope là!

100 - dévoiler (rendre présent) ce qui n'est pas destiné à l'être: dire un secret, ou ce qui pourrait ne pas l'être : moij'aime dire les choses (plutôt que de les penser par derrière) ;

101 - « exprimer » quelque chose à quelqu'un :je te dis toute mon amitié;

102 - effectuer une « mise en scène » ou une « théâtralisation » de cette présentation. : Je te dis : va te faire voir; Je vais le dire à maman que tu m'as tapé; Ça va barder, c'est moi qui te le dis; Dire qu'il faisait si beau ce matin! Je le dis haut et fort: Je ne laisserai pas faire cela. Tu veux que je te dise :j'en ai par-dessus la tête. Il est n'est pas prêt de recommencer, je vais te dire.

103 - constituer une prétérition: il s'agit de formes verbales dont il est dit qu'elles se présentent d'elles-mêmes sans qu'il soit nécessaire qu'un sujet énonciateur les présente. Ce mode de présentation peut renvoyer à des formes d'évidence :

104 Inutile de (te) dire; Il va sans dire que je n'irai pour rien au monde; C'est pas pour dire, mais il exagère, quand même.

105 - prononcer : (auscultation) Ouvrez la bouche et dites «A- $a$ »!

106 Dans tous ces exemples, l'instance qui présente, correspondant à $C_{o}$, renvoie à un sujet énonciateur $\mathrm{S}_{\mathrm{o}}$, mais d'autres instances de présentation sont possibles : voyons ce que dit la météo, le thermomètre, ce texte, cette circulaire, etc. Il s'agit notamment de termes qui présentent la conversion en une forme appropriable (Rf) de données préconstruites. Ces exemples constituent une illustration supplémentaire du fait que dire n'est pas par luimême un verbe de "transmission de paroles". Tous ces emplois correspondent à une coïncidence entre Rf et Rs.

\subsubsection{Rf est construite par l'instance qui présente}

107 C'est l'instance qui présente qui élabore $\mathrm{Rf}$. Rf correspond à une valeur référentielle: en disant ce qu'il dit, $\mathrm{S}_{0}$ parle de quelque chose. Cela signifie que $\mathrm{Rf}$ réfère à un état de choses relativement auquel différents points de vue sont possibles, Rf et Rs entrant alors dans de possibles écarts entre ce qui est présenté et la représentation par $\mathrm{S}$ de ce qui est présenté ( $\mathrm{S}$ pouvant coïncider avec $\mathrm{S}_{\mathrm{o}}$ ). Rs peut correspondre à ce que $\mathrm{S}$ comprend de l'état de choses en question. Je vais te dire ce qui s'est passé: [...] ; Je (te) dis la vérité (à propos d'un état de choses). La vérité en question est celle que je dis, c'est-à-dire telle que je la présente et Rs peut être constituée comme un autre point de vue relativement à ce dont il s'agit avec Rf. 
108 Ces écarts sont le lieu possible d'ajustements, de discordances, de relances, de reformulations. C'est à ce cas que correspond la séquence: Je vois ce que tu veux dire analysée infra. C'est également le cas de nombreuses expressions associées à une formulation qui se cherche. Citons parmi beaucoup d'autres :

109 - comment dire... ponctuant une expression en voie de stabilisation et relevant d'une potentielle reformulation : Il est... comment dire... un peu décalé. L'enjeu est celui d'un ajustement. Comment dire introduit une forme Rf (il est un peu décalé) qui fait l'objet d'une représentation Rs dont la séquence comment dire marque que relativement à Rs d'autres formes sont possibles, ouvrant à d'éventuelles autres représentations R's ;

110 - Disons qui peut correspondre à la cherche d'une forme adéquate relativement à une valeur référentielle ou qui peut encore introduire une forme susceptible de stabiliser une incertitude en sélectionnant un élément dans une classe préconstruite (on peut se voir disons mardi) ;

111 - J'allais dire: Il est, j'allais dire, un peu décalé: la présentation de la forme un peu décalé, laisse ouverte une alternative avec d'autres formes faisant l'objet de représentations R's apparentées.

112 Dans ces exemples où Rf est construite par l'instance qui présente et dont l'enjeu est un ajustement qualitatif entre Rf, Rs et R's, l'instance $S$ n'est en général pas représentée par un allocutaire explicité. Ils doivent être bien distingués de leurs homologues avec présence possible d'un allocutaire correspondant à $\mathrm{S}$, dans lesquels Rf est au contraire préconstruit (cf. le paragraphe précédent) et dont l'enjeu est non pas celui d'un ajustement qualitatif, mais celui de la présentation de Rf préconstruit : on distinguera de ce point de vue a) Je ne sais comment (lui) dire (je ne trouve pas mes mots, dans un effort à élaborer Rf) et b) Je ne sais comment lui dire, dans l'interprétation où dire est proche de annoncer, avec une pondération sur la présentation de Rf préconstruit (je ne sais comment m'y prendre pour lui présenter) ; ou encore J'allais (justement) te le dire ou J'allais (justement)le dire s'adressant à quelqu'un qui a « soufflé la parole » ${ }^{15}$.

113 Ces distinctions font apparaître l'importance d'un troisième facteur de variation concernant la présence ou non d'un allocutaire instanciant le paramètre $S$.

\subsubsection{Présence nécessaire / possible / contrainte d'un allocutaire (correspondant à}

S)

114 La troisième variation concerne la présence ou non d'un terme correspond à $\mathrm{S}$. Cette présence peut être nécessaire (quelque chose me dit qu'il va venir ce soir ; je te dis mon amitié) ; possible (je vais (te) dire ce qui s'est passé; je (te) dis des bêtises) ; ou impossible ou fortement contrainte (cela va sans dire, dire la messe) :

\subsubsection{Présence nécessaire}

115 L'enjeu de la présentation est

116 - soit la construction de S comme site de Rs (§ 4.2.1.1.1.)

117 Quelque chose me dit qu'il va venir ce soir

118 Ce visage me dit quelque chose

119 Ça te dit de venir faire un tour?

120 - soit le ciblage de S comme destinataire singulier de cette présentation: 
Ça va chauffer, je te le dis.

Mais vas-y, jte dis!

Je te dis merci, je te dis va te faire voir.

Je vais te dire une chose:[....]

Je vais te dire ce qui s'est passé.

\subsubsection{Présence possible}

C'est le fait même de la présentation qui est mis en avant sans ciblage particulier sur un $\mathrm{S}$ singulier. Il s'agit d'une présentation qui rend publique une forme, avec la simple possibilité que $\mathrm{S}$ corresponde à un sujet particulier : Paul ( $\left.m^{\prime}\right)$ a dit qu'il reviendrait; je (te) dis des bêtises; inutile de (te) dire que je n'étais pas content; comment (te) dire?

\subsubsection{Présence contrainte ou impossible}

C'est le fait de la présentation qui est mis en avant indépendamment de tout $\mathrm{S}$ singulier.

Cela va sans dire; dire la messe, on va (le) dire (comme ça).

Comme nous l'avons vu dans le paragraphe précédent, la présence ou l'absence d'un allocutaire correspondant à $\mathrm{S}$ peut renvoyer à des cas différents (comme l'a montré la comparaison entre j'allais dire et j'allais te (le) dire.

\section{JE VOIS CE QUE TU VEUX DIRE}

Le tour Je vois ce que tu veux dire présente plusieurs particularités relevant du fonctionnement du verbe dire associé à celui du verbe voir et du verbe vouloir.

Pour comprendre l'enjeu de ces particularités, un détour par le verbe voir est nécessaire, d'autant que voir et dire présentent des affinités en ce qu'ils mettent tous deux en jeu des rapports entre présentation et représentation d'une entité ainsi d'ailleurs, comme nous l'avons vu, que le nom forme. Une donnée qui reste à expliquer est qu'à côté du caractère très naturel et la haute fréquence d'emploi de ce tour, la séquence Je vois ce que tu dis est bien plus rarement attestée, voire perçue comme étrange. Un certain blocage semble donc se produire, et il paraît intéressant, pour en comprendre les raisons, de manipuler la séquence pour observer les déblocages possibles. Des variantes de cette séquence la rendent en effet plus naturelle : avec d'autres verbes que voir : je comprends ce que tu dis, j' entends bien ce que tu dis (en général pour introduire une objection); mais aussi avec des modulations du verbe dire : je vois (ou mieux encore je crois voir) ce que tu essayes de (me) dire, ce que tu es en train de dire.

Enfin, le verbe vouloir entretient lui-même des liens particuliers avec le verbe dire, vouloir dire se trouvant correspondre à différentes valeurs de reformulation. On observe de plus que le verbe dire est à peu près le seul possible dans le tour affirmatif ça veut (ça veut dire qu'il va falloir annuler la cérémonie), alors que toutes sortes de verbes sont possibles à la forme négative (ça ne veut pas partir, démarrer, passer, etc.). Il convient donc d'analyser aussi le rôle de ce verbe dans cette intrication. 


\subsection{Voir}

\subsubsection{Caractérisation de voir}

Voir marque que $\mathrm{S}$ est site en un $\mathrm{t}$ donné d'une représentation $\mathrm{R}$ dont une entité $\mathrm{X}$ est l'objet en se présentant à $\mathrm{S}$ en un $\mathrm{t}$ donné

$\mathrm{S}$ correspond au sujet syntaxique (Co) de voir.

Commentaires de cette caractérisation :

1) Cette caractérisation fait apparaître des affinités avec dire. Ces deux verbes ont en commun d'impliquer la présentation de $\mathrm{X}$ et sa représentation pour un sujet donné. Ils diffèrent sur trois points essentiels :

a) avec dire, l'entité qui fait l'objet d'une représentation est une forme (représentation de référence pour la représentation dont $\mathrm{S}$ est le site ${ }^{16}$;

b) avec voir, $S$ correspond à $C_{0}$. Ce point essentiel signifie pour faire court que le verbe voir implique un sujet qui voit, alors que dire n'implique pas nécessairement un sujet qui dit ;

c) voir met en avant le fait que $S$ est constitué comme site d'une représentation; alors que dire met en avant le fait que $\mathrm{X}$ fait l'objet d'une représentation.

2) Ce qui est vu est à la fois extérieur au sujet qui voit et propre au sujet qui est le site de la représentation ${ }^{17}$, comme le met en évidence l'ambivalence du nominal vue, renvoyant soit à ce qui se présente $(\mathrm{X})$, un "spectacle » : une belle vue; soit à une acuité visuelle ou intellectuelle de S: une bonne vue (sans lunettes); une bonne vue d'ensemble du problème.

3) Voir est un événement lié à ce qui se présente à un moment donné (contrairement à regarder ou entendre: Par oiseau, j'entends un oiseau qui chante.... / ?? Par oiseau, je vois...).

4) Représentation peut renvoyer à différentes activités mentales telles que compréhension (Je ne comprends pas tout, mais je vois en gros l'idée; C'était un peu comme un frère pour moi, tu vois...) ; appropriation d'une perception sensorielle ( $\mathrm{Tu}$ as vu comme le vent nous a poussés!) ; constat (Tu vois, ça n'était pas si douloureux, finalement) ; interprétation ( Dans cette attitude, je vois plutôt une forme de lâcheté; On peut voir du Proust dans cette évocation); image mentale (je vois une fumée noire s'élever à l'horizon); attestation de la réalité (Je l'ai vu de mes yeux vu); évocation (Ah oui, je vois de qui tu veux parler); appropriation d'une idée (Je ne vois pas pourquoi c'est moi qui irais), etc. Ce qu'on isole sous forme de "perception visuelle», au risque d'y cantonner ce verbe, ne correspond qu'à un cas particulier (comparer: $\mathrm{Tu}$ as vu cette fleur / Tu as vu comme elle est belle, cette fleur, tu as vu la beauté de cette fleur?)

\subsubsection{Variation de voir}

\subsubsection{Centrage sur la mise en présence de $X$}

Il y a centrage sur le fait que $\mathrm{X}$ se présente à $\mathrm{S}$ (sur l'entité vue). Centrage QNT.

\subsection{X préconstruit}

$\mathrm{X}$ est préconstruit indépendamment de sa mise en présence. L'existence de $\mathrm{X}$ est dissociée de sa présence. $X$ préconstruit passe de non présent à présent : 
146 - Un taxi,j'en vois un justement.

147 Il est question de $\mathrm{X}$ indépendamment de sa mise en présence. $\mathrm{R}$ est de l'ordre de voir pour autant que $\mathrm{X}$ se présente à $\mathrm{S}$ (indépendamment de voir, et en tant qu'espéré ou imaginé, $\mathrm{X}$ peut faire l'objet d'autres types de représentation que celle mise en jeu par voir qui implique mise en présence de $\mathrm{X}$ ).

148 - Oh tu sais, des incidents de ce genre, on en voit tous les jours (c'est le propre de ces incidents d'être visibles) = Il s'en présente tous les jours (en tant que faisant objet de R pour S)

149 - Le fantôme, je l'ai vu, je n'ai pas rêvé. Il s'est bel et bien présenté à moi.

150 - Tu les vois, les traces? - Ah ça y est, je les vois! (elles se présentent à moi).

151 - Tu as vu le dernier film de Tarentino?

152 - Tu as réussi à voir son visage?

$153-V u !$

\subsection{X non préconstruit} réaliser, imaginer, prendre conscience de, envisager, etc.)

\subsection{Présence de X préconstruite}




\subsection{Présence de $X$ non préconstruite} vouloir bien repérables dans le cas où $\mathrm{S}=j e\left(\mathrm{C}_{\mathrm{o}}\right)$ au présent de l'indicatif (je veux...). On relève par exemple qu'un exemple comme je veux dormir implique un contexte du type: laisse-moi tranquille (présence d'une gêne) ou encore que la forme je veux partir implique que par ailleurs partir est construit comme non validable, a étant associé à un empêchement, un refus ou une interdiction $\left(\mathbf{a}=\mathbf{S}^{\prime}\right)$. On observe que cette séquence est nettement plus naturelle dès lors qu'est mentionnée une résistance ou un obstacle : mais c'est que moi je veux absolument y aller! La forme elle aussi plus naturelle du conditionnel est assortie de la mention d'une contrariété : je voudrais partir, mais cela s'avère hélas peu réaliste.

La mise en présence de $\mathrm{X}$ correspond à une façon de voir X.

Grâce à vos questions, je commence à voir ce qui m'était invisible. prend le statut d'antérieurement invisible. Ce qui m'était invisible n'a de statut qu'à travers sa représentation naissante (le fait de commencer à le voir). Ce qui m'était invisible devient présent dans la mesure où j'en ai une représentation ${ }^{18}$.

\subsection{Vouloir}

(2017).

1) qu'un procès $\mathbf{q}$ fait l'objet d'une double construction relative à deux sites de construction disjoints :

- un site a (disjoint de $\mathbf{S}$ ) où $\mathbf{q}$ est construit comme validable (validation de $\mathbf{q}$ non entérinée);

2) q ne peut être construit en même temps comme validable (relativement à a) et validé (relativement à S).

quant à la réalisation / validation de $\mathbf{q}$

disjonction alternative : ou bien pour $\mathbf{q}$ validé en $\mathbf{S}$, $\mathbf{q}$ est en même temps non validable en $\mathbf{a}$; ou bien pour $\mathbf{q}$ validable en $\mathbf{a}, \mathbf{q}$ ne peut être validé en $\mathbf{S}$ que dans un temps distinct.

La forme je veux bien est en revanche parfaitement naturelle. Elle marque une dissociation temporelle entre la construction de $\mathbf{q}$ comme validable (proposition) et comme validée (proposition entérinée).

3 Cette caractérisation rend compte également des cas où la forme négative est obligatoire. C'est le cas avec les exemples Le moteur ne veut pas démarrer (mais ?? Le moteur veut démarrer); Le café ne veut pas passer. Cette tache ne veut pas partir. Le sommeil ne veut pas venir.

Dans ces exemples, la positivité de $\mathbf{q}$ est établie par sa construction comme validé en $\mathbf{S}$. Vouloir marque alors que q est nécessairement construit comme non validable dans le 
même temps relativement à a, ce que marque la forme négative obligatoire de vouloir. Relativement à $\mathbf{S}$, sommeil venir est validé ; relativement à a (sommeil), venir n'est pas validable. Il s'agit d'un cas où $\mathrm{C}_{\mathrm{o}}$ correspond à $\mathbf{a}$.

Enfin, la temporalité mise en avant dans cette caractérisation est clairement illustrée dans les exemples: Quand tu veux! L'action est à la fois validée et validable (par le destinataire de cette adresse) dans un temps distinct. On notera la singularité de cette séquence qui n'admet que le verbe vouloir. Contexte : on n'attend plus que toi. $\mathbf{S}^{\prime}=\mathbf{a}$ (correspondant ici à l'énonciateur $\mathrm{S}_{\mathrm{o}}$ de l'énoncé) construit $\mathbf{q}$ (faire ce dont il s'agit) comme validable relativement au fait que $\mathbf{q}$ n'est pas (encore) validé relativement à $\mathbf{S}$. La validation effective de $\mathbf{q}$ ne dépend que de sa construction dans la position $\mathbf{S}$ : il ne tient qu'au sujet correspondant à $\mathbf{S}(t u)$ de rendre $\mathbf{q}$ effectif à tout moment, quand introduisant une dimension temporelle. Relèvent également de ce cas deux types d'emplois: 1) proposition: On peut y aller demain, si tu veux ; 2) ponctuant d'un discours (si tu veux est alors souvent proche de en quelque sorte) : C'était un peu son deuxième père, si tu veux... C'est l'énonciateur qui construit $\mathbf{q}$ (la proposition c'était un peu son deuxième père) comme validable, l'interlocuteur correspondant à $\mathbf{S}$ prenant le statut de valideur conditionnel et passif (emploi rhétorique).

L'affinité entre vouloir et dire tient à ce que chacun de ces deux verbes met en jeu une dualité de sites.

\subsection{VOULOIR DIRE}

Il convient de ne pas confondre deux types d'emplois bien distincts de vouloir dire

a) emplois caractérisés par les critères suivants :

- dire met en jeu un $\mathbf{S}$ allocutaire (explicite ou non);

- vouloir dire relève d'une composition immédiatement intelligible de vouloir et de dire : dire correspond à q (complément direct de vouloir) ;

- vouloir ainsi que dire sont remplaçables par une série d'autres verbes : je veux (tiens à, me fais un devoir de, me dois de, souhaite, etc.) te dire (déclarer, prévenir, faire savoir, informer, etc.) clairement que les choses ne peuvent continuer ainsi ; ce que je veux dire aux Français, c'est que la peur n'est pas bonne conseillère; voilà ce que je voulais vous dire...

- vouloir est associé à une intention ciblée de l'énonciateur sujet de dire.

On distingue deux cas :

194 - emplois de je veux (première personne du présent de l'indicatif), relativement contraints pour les raisons précédemment mises en avant avec l'analyse de vouloir.

195 - autres emplois :

196 Que voulais-je (te) dire? (j'avais l'intention de dire quelque chose ; j'ai oublié où j'en étais).

Qu'a-t-il voulu (eu l'intention, cherché à) dire?

b) emplois où vouloir dire exclut un complément attributaire et où ni dire ni vouloir ne peut guère être remplacé par un autre verbe. Vouloir dire apparait alors comme un bloc figé, mais dans lequel nous défendons l'hypothèse que ces deux verbes conservent leur pleine valeur sémantique. 
Soulignons à cet égard que dire est le seul verbe possible dans le tour ça veut à la forme affirmative ${ }^{19}$, alors que toutes sortes de verbes sont possibles à la forme négative : ça ne veut pas démarrer, pas marcher, pas venir, etc.).

Dans ces emplois, dire ne renvoie pas à la présentation d'une forme préconstruite dont vouloir marquerait quelque intention de la présenter, mais correspond à différents cas de reformulation. Vouloir dire signifie que la présentation d'une forme $\mathrm{Rf}$ en faisant de $\mathrm{S}$ le site de Rs (validée du fait même de la présentation de Rf) marque que cette même forme rend validable la construction d'une autre représentation R's, dans des rapports d'altérité variables à Rs.

L'altérité tend vers une identification dans un exemple comme: le mot meek veut dire humble en anglais; vers une altérité forte dans les cas où vouloir dire correspond à une rectification: On se voit donc mardi, euh! lundi j'veux dire ${ }^{20}$; vers une altérité forte doublée d'une identification dans le cas où l'instance qui présente correspond à ça : La lune est brouillée, ça veut dire qu'il va pleuvoir. Rappelons (cf. § 4.2.1.1.3.) que ça renvoie à l'actualisation, dans le cadre de l'énonciation, de la mention de ce dont il s'agit dans toutes ses composantes en bloc, sans ancrage situationnel. Ça confère à ce à quoi il renvoie un mode de présence qui n'a d'autre détermination que celle que lui confère l'énonciation dans son actualisation. Rf (la lune est brouillée) est validé du fait de sa présentation par l'instance ça et vouloir maque que R'f (autre que Rf : il va pleuvoir) est validable. Rf et R'f sont identifiables (modulo le passage par ça) tout en étant dans un rapport d'altérité forte.

\subsection{Contrainte sur Je vois ce que tu dis}

202 Le caractère peu naturel de la séquence je vois ce que tu dis peut être mis en regard de l'exemple : ce visage me dit quelque chose. La glose en serait (en assignant $m e$ à $\mathrm{S}$ ) : je vois ce que me dit ce que tu dis, et ce que tu me dis me dit quelque chose. Cette glose marque la construction (QNT) de S (me) comme site d'une représentation Rs dont une forme (ce visage / ce que tu dis) est l'objet. Nous avons montré que cette construction QNT correspond à une opacité QLT. S ne peut être à la fois et dans le même temps construit comme site d'une $\mathrm{R}$ dont une forme est l'objet en se présentant et déterminer en même temps en quoi consiste qualitativement cette forme ${ }^{21}$.

203 Il est donc nécessaire de dissocier construction QNT de S comme site de Rs et détermination QLT de cette Rs. C'est ce que permet une modalisation telle que : je vois ce que tu essayes de (me) dire qui introduit une confrontation entre deux (ou plusieurs) déterminations QLT de Rs, différentes façons de « comprendre » ce que tu dis. Le candidat le plus naturel à cette dissociation est le verbe vouloir, dont nous allons voir pourquoi il entretient des liens particuliers avec le verbe dire.

\subsection{Intrication de voir, vouloir et dire dans je vois ce que tu veux dire}

A la suite des caractérisations précédentes une désintrication des trois verbes voir, vouloir et dire apparait possible.

- Je vois ce que tu veux dire peut-être glosé par Je vois ce que tu veux dire en disant ce que tu dis. Ce que tu dis correspond à Rf qui est validé du fait de sa présentation ; 
- Je ne peux voir ce que tu dis dans le présent de sa présentation pour les raisons exposées : lorsque je vois une forme dans le présent de sa présentation, la détermination qualitative de la Rs dont elle fait l'objet est réduite au seul fait que cette forme se présente (la forme que je vois n'est autre que la forme de ce dont elle est la forme) ;

207 - l'introduction de vouloir avec la mise en jeu de ses deux repères signifie que ce que tu établis en disant ce que tu dis (la valeur référentielle, ce dont il s'agit), validé du fait que tu le dis, est aussi un point de vue sur ce que tu dis (sur cette valeur référentielle), une façon de dire ce dont il s'agit, qui rend validable un autre point de vue sur ce dont il s'agit, engageant une autre façon de dire possible.

Je vois ce dont il s'agit à travers ce que tu dis, d'un point de vue ouvert par celui auquel correspond ce que tu dis, mais en décalage par rapport à lui.

Cette décomposition analytique peut se reformuler de façon plus synthétique et plus directement interprétable: Je vois ce que tu veux dire signifie que je vois autre chose que ce que tu dis en disant ce que tu dis (que je ne peux pas voir). Ce que tu dis est la condition pour que je puisse voir ce que tu dis, au prix d'un déplacement par lequel je ne peux voir (me représenter) qu'autre chose que ce que tu dis (relativement à ce dont il s'agit à travers ce que tu dis). Ce que tu veux dire relève de mon «interprétation» ou d'un point de vue parmi d'autres possibles sur ce dont il s'agit à travers ce que tu dis.

Vouloir dire ici ne relève en rien d'une intention de dire, mais d'une interprétation qui se dit dans un rapport d'altérité à ce qui est effectivement dit et qui peut se traduire par une reformulation.

\section{Conclusion}

211 Notre analyse a permis d'une part de montrer que dire n'est pas en soi un verbe de parole (Ce visage me dit quelque chose; Ça te dit de venir faire un tour? Voyons ce que dit le thermomètre ); que vouloir n'est pas un verbe de « volonté » ni d'intention (Je ne sais pas ce que veut dire ce mot ; C'était un peu son deuxième père, si tu veux...) ; que voir n'est pas un verbe de perception visuelle ( $\mathrm{Tu}$ as vu comme le vent nous a poussés ?! Je n'ai pas tout compris, mais je vois l'idée en gros), mais qu'une caractérisation de ces verbes rendant compte de ces emplois permet d'expliquer la façon dont ils peuvent, à certaines conditions, prendre ces valeurs ; d'autre part de montrer que dans la question $\mathrm{Tu}$ vois ce que je veux dire? les trois verbes en jeu conservent leur pleine identité, avec une intrication des paramètres constitutifs de cette identité.

\section{BIBLIOGRAPHIE}

Camus, R. \& De Vogüé, S. (eds), 2006, Variation sémantique et syntaxique des unités lexicales, étude de six verbes français, LINX, 50.

Franckel, J.-J. \& Lebaud, D. 2006, «Forme », in Antoine Culioli, Un homme dans le langage, colloque de Cerisy, Dominique Ducard et Claudine Normand (eds), Ophrys, p. 332-357. 
Franckel, J.-J. ; 2015, dire in « Dire et ses marqueurs », Langue Française, 186, 2/2015, Larousse, p.87-102.

Franckel, J.-J. , 2017, « Vouloir dire », (2017) in Claudine Normand, une vie dans le langage, Driss Ablali et Frédérique Sitri (eds), LINX, 74, p.39-66.

Gomez-Jordana, S. \& Anscombre, J.-C., 2015, « Dire et ses marqueurs », Langue Française, 186, 2/2015, Larousse.

Jalenques, P., 2002, «Étude sémantique du préfixe RE en français contemporain : à propos de plusieurs débats actuels en morphologie dérivationnelle », Langue française, Volume 133 - numéro 1, p. $74-90$.

Nicolaev, A., (2012) «The Story of Latin dīcere », Oxford University, 23 February 2012, en ligne.

Paillard, D., 2006, « À propos des verbes préfixés avec la base KAZ- en russe contemporain », in Lebaud D., Paulin C., Ploog K. (éds), Constructions verbales et production de sens, Recherches en Linguistique Étrangère XXIV, Presses Universitaires de Franche-Comté, 2006, p. 321-336.

Rouanne, L. \& Anscombre J.-C. (eds), 2016, Histoires de dire, Peter Lang.

\section{NOTES}

1. Cet article s'inscrit dans l'espace théorique ouvert par Antoine Culioli. Je remercie les membres du séminaire TOPE (théorie des opérations prédicatives et énonciatives) pour leurs questions et remarques sur une première version de ce texte, et tout particulièrement Rémi Camus à qui je dois plusieurs ajouts, références, exemples et remarques de pénétrante sagacité.

2. Dans les descriptions (recouvrant la quasi-totalité de celles qui lui sont consacrées) qui tiennent dire comme un verbe «de parole ", ces emplois posent problème et sont traités comme relevant d'extensions métaphoriques. Dans notre approche, ces emplois (dont on ne voit pas au juste en quoi et de quoi ils seraient des métaphores) sont au contraire fondamentaux et au plus proche de l'identité du verbe. C'est lorsque dire est associé à un environnement d'ordre verbal (par exemple Il me dit des mots d'amour) que dire est, par « contamination contextuelle», perçu comme « verbe de parole ", ce qu'en réalité il n'est pas par lui-même.

3. Cette caractérisation se présente comme une «forme schématique ». Celle-ci est composée de paramètres auxquels peuvent correspondre différentes unités du co-texte selon sa structure syntaxique, et vise à rendre compte de la façon dont une unité morpho-lexicale peut formater son co-texte et son contexte et construire ses valeurs. Elle appréhende l'unité dans une dimension discursive. Sur cette notion, cf. en particulier Camus et De Vogüé (2006). La formulation proposée ici diffère sensiblement de celle présentée par l'auteur dans de précédents articles (Franckel (2015) et (2017)).

4. La présentation par $\mathrm{S}_{\mathrm{o}}$ d'une forme à un sujet $\mathrm{S}$ fait écho à l'étymologie du verbe dire qui remonte à la racine indo-européenne *deik- / *dik. renvoyant à l'acte de montrer, de désigner, de donner à voir, d'attirer l'attention. Pour une étude approfondie de cette étymologie, cf. en particulier Nicolaev (2012). Pour Levinas dans Le visage de l'autre, «l'acte de désigner place les choses dans la perspective d'autrui ".

5. Pour une présentation et une discussion des études sur ce préfixe, cf. en particulier Jalenques (2006)

6. Le terme de représentation tel qu'introduit ici peut faire écho à celui de « représentification » au centre de textes traitant de la métaphysique du présent. Le terme de représentation que nous réduisons dans cette analyse linguistique à ses rapports avec celui de présentation fait l'objet 
d'une vaste littérature en particulier dans le domaine de la philosophie. Nous laissons de côté ici les emplois de représentation à valeur itérative comme dans le cas de représentations d'un spectacle.

7. Merleau-Ponty (dans Le visible et l'invisible) parle de la distance constitutive entre ce qui se présente (de ce qui paraît) et ce qui se représente.

«Le propre de ce qui paraît est de se donner dans une profondeur irréductible, une sorte de distance qui n'est pas l'envers d'une proximité possible. La chose paraissante est transcendante, non pas au sens d'un objet situé dans l'extériorité, à une distance en droit réductible, mais en ceci que la transcendance fait son être. La distance du perçu n'en est pas un trait extrinsèque. Le perçu ne peut être posé hors de sa distance car elle pour ainsi dire la forme de son apparaitre : la chose n'est pas là-bas parce qu'elle est à distance de moi, elle est au contraire à distance de moi parce qu'elle est là, parce qu'elle apparaît. »

C. Rosset :

«Privée d'immédiateté, la réalité humaine est également privée de présent. Ce qui signifie que l'homme est privé de réalité tout court, si l'on en croit là-dessus les stoïciens dont un des points forts fut d'affirmer que la réalité se conjuguait au seul présent. Mais le présent serait par trop inquiétant s'il n'était qu'immédiat et premier: il n'est abordable que le biais de la représentation, selon une structure énonciative qui l'assimile à un passé ou à un futur, à la faveur d'un léger décalage qui en érode l'insoutenable vigueur et n'en permet l'assimilation que sous les espèces d'un double plus digeste que l'original dans sa crudité première. »

«Si le double parvient, comme il y parvient en effet de manière incomparable, à " représenter » le réel, c'est justement parce qu'il contredit toute possibilité de représentation et réussit ainsi la performance de présenter le réel en tant que non représentable ".

8. La formulation de cette caractérisation ainsi que l'analyse proposée diffère sensiblement de celle donnée par Franckel et Lebaud (2006).

9. Il s'agit d'ajustements qualitatifs dont la reformulation n'est que l'un des cas possibles. On peut par exemple mentionner la séquence On peut dire ça comme ça, qui met en jeu une articulation entre $c ̧ a_{1}$ et $c a_{2}$. Ça $a_{1}$ correspond à une Rs dont Rf peut constituer une référence possible. D'autres exemples sont donnés en 4.2.3.3.

10. On notera la parenté de sens entre cet énoncé et Je sens qu'il va venir ce soir. Cette proximité résulte de fonctionnements bien différents (cf. la caractérisation de sentir donnée au $§$ 2.1.3.).

11. Rf n'a de statut que dans le présent de sa présentation, ce qui explique que cet emploi n'est possible qu'au présent ou à l'imparfait. Rémi Camus (communication personnelle) fait remarquer l'ambivalence du "petit doigt » : au présent (mon petit doigt me dit que tu n'as pas fait tes devoirs), mon petit doigt peut être assimilé au "quelque chose »; au passé composé on passe à un emploi dialogique, mon petit doigt se substituant à quelque mystérieux énonciateur.

12. Dire quelque chose dans ce cas contraste singulièrement avec parler: cette histoire me parle signifie qu'elle ne me laisse pas indifférent, stimule une réaction.

13. Il peut également s'agir du négatif, dans le cas de Ça ne me dit rien analysé dans le § suivant, ainsi que des emplois au conditionnel : Ça me dirait bien de partir avec toi.

14. Je dois ces beaux exemples à Rémi Camus

15. L'examen des expressions recensées par Rouanne \& Anscombre (2016), p.366-369 tend à montrer qu'une répartition grossière est possible entre expressions relevant d'une préconstruction de Rf avec pondération sur la présentation d'une part et expressions sans préconstruction de $\mathrm{Rf}$ et relevant d'un ajustement qualitatif entre $\mathrm{Rf}$ et Rs, d'autre part.. Des expressions telles que soit dit en passant, vous me direz (m'objecterez, me ferez valoir), je me suis laissé dire, comme qui dirait, disons-le, faut dire, c'est juste histoire de dire semblent relever du premier cas ; en revanche, disons, autrement dit, pour ainsi dire, je dirai... du second. Resterait à voir comment les outils proposés dans cet article permettraient de rendre compte de ces expressions sinon dans la finesse de leur description, du moins dans le principe de leur mécanisme. 
16. Ce point permet d'expliquer pourquoi à côté de Je vois une tache, la séquence je dis une tache est fortement contrainte (je dis (prononce) : « une tache »; on dit une tache et non un pâté). Cette contrainte doit être mise en regard de on dirait une tache. Comme suggéré supra avec on dirait un lion, une tache / un lion est l'identification déplacée / déformée du quelque chose identifiable mais non identifié qui se présente. La contrainte touche à la variation Rf préconstruite ou non. Si Rf est préconstruite, c'est déjà en soi une forme (une vérité dans je dis une vérité); si elle n'est pas préconstruite, on retrouve le quelque chose dont la tache n'est que cette identification hasardeuse ( on dirait). La parenté entre voir et dire peut être illustrée par l'exemple : (s'agissant du style d'un texte) On dirait du Proust proche de : Dans le style de ce texte, on peut voir du Proust.

17. Wittgenstein note plaisamment qu'« on écarquille les yeux pour voir, on les ferme pour se représenter ».

18. L'adjectif visible est formé avec le suffixe -ible qui marque que la $\mathrm{R}$ dont $\mathrm{X}$ est l'objet n'est pas nécessairement actualisée. D'autre part, $\mathrm{S}$ est un site indéterminé : $\mathrm{X}$ est visible pour / par qui (quiconque) peut / veut voir X. De même que voir est toujours événementiel, circonstanciel, visible est une propriété circonstancielle de X (il ne peut s'agir d'une propriété inhérente). On peut avoir sa fatigue est visible (fatigue circonstancielle), alors que sa barbe est visible est contraint et met en jeu d'une temporalité : la barbe de ce jeune adolescent commence à être visible; sur ce cliché, la barbe est nettement visible. Comparer de même ?? Il était visiblement africain // Il était visiblement épuisé. Les variations de visible sont calquées sur celles de voir: centrage sur $\mathrm{X}$ avec $\mathrm{X}$ non préconstruit ou préconstruit, ou centrage sur R. Avec le centrage sur X préconstruit, visible correspond à " présentable " (Madame la comtesse est visible; Ces tableaux sont visibles dans la galerie Z. Ces tableaux seront visibles du 2 février au 21 mars); avec centrage sur X non préconstruit, l'existence de $\mathrm{X}$ est non dissociée de sa mise en présence qui le constitue comme objet possible de $\mathrm{R}$; visible marque que $\mathrm{X}$ peut faire l'objet d'une $\mathrm{R}$ établissant la mise en présence et partant, l'existence de $\mathrm{X}$ en tant qu'objet de cet objet possible de $\mathrm{R}$ (Son émotion était visible; on la voit = on peut la voir, et de ce fait elle est visible et donc présente. Son émotion acquiert un mode d'existence du fait qu'on (S indéterminé) les voit et de ce fait peut les voir (X est visible pour peu qu'un $\mathrm{S}$ donné voie $\mathrm{X}$ ). Avec le centrage sur $\mathrm{R}$, visible est associé à la façon dont $\mathrm{S}$ (indéterminé) peut voir $\mathrm{X}$, avec deux cas : 1) X est construit comme destiné à être vu (Les panneaux sont bien visibles : le caractère visible des panneaux est circonstanciel : on les voit bien, sont bien visibles pour ceux à qui ils se présentent) ; 2) le cas où le caractère visible de $\mathrm{X}$ tient au fait qu'on peut voir X à un moment donné (Le mont St-Michel commence à être visible au loin. Sous cet angle, les traces sont à peine visibles). On peut distinguer son émotion était visible qui confère un statut d'existence à son émotion et Son émotion était à peine visible relevant du présent cas, où l'existence de son émotion n'est pas en jeu et où seule est prise en compte la façon dont son émotion peut être vue. Le premier cas marque qu'il y a de l'émotion; le deuxième cas établit, étant donné la présence d'émotion, comment on la voit.

19. Cette remarque ne vaut que pour ça «impersonnel », sans prise en compte des cas où ça désigne de façon péjorative une entité humaine (ces gens là, ça se croit tout permis), ni de certains régionalismes (Ça veut pleuvoir) où de plus peut jouer une assonance entre veut et va.

20. Dans ces emplois, mais plus largement dans les reformulations dont le $C_{o}$ est je, je veux dire se transforme en j'veux dire.

21. Cette opacité qualitative peut être mise en regard, du point de vue des variations du mot forme, du cas je vois une forme qui apparaît au loin / se détacher contre le mur, (cf. 3.2.2.3), où c'est le fait même qu'elle se présente qui constitue la seule détermination qualitative de forme: il est impossible de déterminer de quoi cette forme est la forme, de donner une caractérisation qualitative de cette forme autre que le fait qu'elle se présente à un moment donné. 


\section{RÉSUMÉS}

Cet article vise à rendre compte de la façon dont les trois verbes composant l'expression Je vois ce que tu veux dire interagissent pour en construire le sens. L'article propose à cet effet une caractérisation de chacun de ces verbes (voir, vouloir et dire) en dégageant leur identité à travers le déploiement de la variation de leurs valeurs. Il est en particulier montré que dire n'est pas en soi un verbe de parole (Ce visage me dit quelque chose; Ça te dit de venir faire un tour? Voyons ce que dit le thermomètre) ; que vouloir n'est pas un verbe de "volonté » ni d'intention (Pourquoi veux-tu que ça rate ; C'était un peu son deuxième père, si tu veux...) ; que voir n'est pas un verbe de perception visuelle ( $\mathrm{Tu}$ as vu comme le vent nous a poussés ?! Je n'ai pas tout compris, mais je vois l'idée en gros), mais qu'une caractérisation de ces verbes rendant compte de ces emplois permet d'expliquer la façon dont ils peuvent, à certaines conditions, prendre ces valeurs. Cette analyse conduit en outre à rendre compte de contraintes significatives, en particulier le caractère peu naturel de la séquence? Tu vois ce que je dis (ou? je vois ce que tu dis); et le fait que dire est seul verbe compatible avec le tour Ça veut + V (à la forme affirmative).

The aim of this article is to show how the three verbs used in the French expression Je vois ce que tu veux dire («I see what you mean ») interact in the construction of its meaning. To achieve this aim, this article proposes a characterization of the verbs voir, vouloir and dire, while working out their identity through the widest possible range of variation of their uses. It particularly shows that dire does not necessarily involve words (Ce visage me dit quelque chose ; Ça te dit de venir faire un tour? Voyons ce que dit le thermomètre) ; that vouloir does not involve intention or will (Pourquoi veux-tu que ça rate? C'était un peu son deuxième père, si tu veux...) ; and that voir does not involve visual perception ( Tu as vu comme le vent nous a poussés ?! Je n'ai pas tout compris, mais je vois l'idée en gros). Such characterization of these verbs nevertheless accounts for the possibility for them to take such meanings, arguing that they do so only under certain conditions. The analysis also makes it possible to account for the fact that a sequence such as Je vois ce que tu dis (or Tu vois ce que je dis?) is not natural and that dire is about the only verb compatible with the sequence ça veut $+\mathrm{V}$.

\section{INDEX}

Mots-clés : dire, voir, vouloir, forme, interaction, construction du sens

Keywords : dire, voir, vouloir, forme, interaction, meaning construction.

\section{AUTEUR}

\section{JEAN-JACQUES FRANCKEL}

Université Nanterre-Paris-ouest- La Défense 